\title{
Synthesis of a Near-Optimal High-Gain Antenna Array with Main Lobe Tilting and Null Filling Using Taguchi Initialized Invasive Weed Optimization
}

\author{
Zaharias D. Zaharis, Pavlos I. Lazaridis, John Cosmas, Senior Member, IEEE, Christos Skeberis, and \\ Thomas D. Xenos
}

\begin{abstract}
A near-optimal base-station antenna array synthesis suitable for broadcasting applications is presented. The array is required to provide a high-gain radiation pattern with a main lobe slightly tilted from the horizontal plane and null filling inside an angular sector under the main lobe. To satisfy the above requirements, a novel Invasive Weed Optimization (IWO) variant called Taguchi Initialized IWO (TI-IWO) is proposed in the present paper. In TI-IWO, the Taguchi's Optimization method is employed to initialize effectively the positions of the weeds used by the IWO method. In this way, the fitness function starts from lower values and thus the TI-IWO method finds better near-optimal solutions than the conventional IWO method. The proposed method has been applied to linear arrays. Due to its easy implementation in practice, a uniform-amplitude excitation distribution is considered to be applied on the array elements. Two cases of isotropic source arrays are studied under specific requirements for maximum possible gain, main lobe tilting and null filling. Also, the TI-IWO method is applied to optimize realistic cases of collinear wire dipole arrays in front of a mast under the same requirements and an additional one concerning the impedance matching of all the dipoles.
\end{abstract}

Index Terms-Antenna array, main lobe tilting, null filling, invasive weed optimization, Taguchi's optimization

\section{INTRODUCTION}

$\mathrm{M}$ ODERN research on antennas has become very challenging, especially in the area of broadcasting [1][3]. A lot of techniques have been proposed for the design of base station antenna arrays in order to satisfy requirements, which are essential for broadcasting applications [4]-[8]. These requirements usually considered by a broadcasting antenna array are given below: (i) Due to the large distance

Manuscript received March 10, 2013.

Zaharias D. Zaharis is with the Telecommunications Centre, Aristotle University of Thessaloniki, 54124 Thessaloniki, Greece (e-mail: zaharis@auth.gr).

Pavlos I. Lazaridis and John Cosmas are with the School of Engineering and Design, Brunel University, London UB8 3PH, U.K. (e-mail: pavloslazaridis@hotmail.com; john.cosmas@brunel.ac.uk).

Christos Skeberis and Thomas D. Xenos are with the Department of Electrical and Computer Engineering, Aristotle University of Thessaloniki, $54124 \quad$ Thessaloniki, Greece (e-mail: cskeberis@auth.gr; tdxenos@eng.auth.gr). between the transmitting base station and the service area, the antenna array needs to produce a very narrow main lobe which, in conjunction with the need for reduction of the spatial spread of radiated power, results in the requirement of maximum gain. (ii) Provided that the broadcasting base station is usually located at higher places relative to the service area, the main lobe is required to be tilted from the horizontal plane. Due to the large distance from the service area, the tilting angle is usually small (i.e., $2^{\circ}$ or $3^{\circ}$ ). (iii) In order to have satisfactory reception of transmitted signal inside an angular sector under the main lobe, the directional gain is not permitted to fall below a certain value in relation to the maximum gain value, which results in filling of radiation pattern nulls inside the above-mentioned angular sector. The level of null filling depends on the service type (e.g., FM radio, DVB-T) and the value of signal-to-noise ratio $(S N R)$. (iv) In order to reduce the power reflection along the feeding lines and thus increase the efficiency of the whole feeding network, the impedance matching condition is required for every array element, which means that the standing wave ratio $(S W R)$ of every element must be close to unity.

It is obvious that the design of such an antenna array is a multi-objective problem, since the above requirements must be simultaneously satisfied. Therefore, an optimization method is necessary to solve this kind of problem. Such an efficient method recently proposed is the Invasive Weed Optimization (IWO) method [9]-[14]. The IWO is an evolutionary method inspired from the invasive nature of weeds. Due to its fast convergence and performance, the IWO has been chosen to solve many problems in the area of electromagnetics. However, the stochastic behavior of the method restricts its convergence and performance.

The present paper introduces a novel modified version of IWO called Taguchi Initialized IWO (TI-IWO). The Taguchi's optimization method is utilized here to initialize effectively the positions of the weeds inside the search space, instead of creating random initial positions as happens in the conventional IWO method. In this way, the fitness function starts from lower values, and thus the TI-IWO method results in lower fitness values than the conventional IWO method.

The proposed method has been applied to optimize linear arrays according to the above-specified requirements. In all 
the cases studied here, a uniform-amplitude excitation distribution is considered to be applied on the array elements, since excitations of equal amplitudes are easily implemented in practice. In the first two cases, linear arrays of 8 and 16 isotropic sources, respectively, are optimized for maximum gain, main lobe tilting and null filling, while the impedance matching condition is not required due to the use of isotropic sources. Also, the TI-IWO method is applied to optimize two realistic cases of collinear wire dipole arrays ( 8 and 16 dipoles respectively) in front of a mast by taking into account the four requirements defined above. The radiation characteristics of each array need to be calculated for every evaluation of the fitness function, which is going to be minimized by the TIIWO method. The radiation characteristics of the wire dipole arrays (including the input impedances of the dipoles) are extracted by applying the Method of Moments (MoM) [15]. The optimization results exhibit the effectiveness of the proposed method.

\section{INVASIVE WEED OPTIMIZATION}

The IWO method has initially been proposed by Mehrabian and Lucas [9]. Due to its fast convergence and performance in comparison to other methods, it has been used to solve several problems of antenna optimization [10]-[14].

The IWO algorithm simulates the colonizing behavior of weeds in nature. Initially, a population of weeds is dispersed at random positions inside an $\mathrm{N}$-dimensional search space, where $N$ is the number of parameters to be optimized by the IWO algorithm for the given problem. These positions are produced by a uniform random number generator. The optimization algorithm is an iterative process and consists of three basic steps repeatedly applied at each $i$-th iteration. These steps are:

\section{Step A: Reproduction}

Each $w$-th weed produces a number of seeds $n s$, which depends linearly on the fitness value of the weed, according to following expression:

$$
\begin{gathered}
n s(w, i)=\operatorname{int}\left[n s_{\min }+\left(n s_{\max }-n s_{\min }\right) \frac{f i t(w, i)-f i t_{\min }(i)}{f i t_{\max }(i)-f i t_{\min }(i)}\right], \\
w=1,2, \ldots, W \quad \& \quad i=0,1,2, \ldots, I
\end{gathered}
$$

where $f i t(w, i)$ is the fitness value of the $w$-th weed at the $i$-th iteration, $f i t_{\text {min }}(i)$ and $f i t_{\text {max }}(i)$ are respectively the minimum and the maximum fitness value at the $i$-th iteration, $n s_{\min }$ and $n s_{\max }$ are the limits of $n s, W$ is the population size, $I$ is the maximum number of iterations, and finally int [-] defines the integer part of a number. The parameters $W, I, n s_{\min }$ and $n s_{\max }$ are defined by the user. Provided that the optimization process aims at minimizing the fitness function, $f i t_{\min }(i)$ and $f i t_{\max }(i)$ are respectively the best and the worst fitness value. Consequently, the "bad" weeds (with high fitness values) are given the possibility to produce more seeds than the "good" weeds (with low fitness values) according to (1). By producing more seeds, a weed is more likely to find positions with better fitness values and thus approach the optimum position.

\section{Step B: Spatial Dispersion}

The seeds produced by every weed are randomly dispersed around the weed. The dispersion is performed according to a normal distribution with standard deviation $\sigma$, which decreases as a function of the number of iterations $i$, according to the expression:

$\sigma(i)=\frac{(I-i)^{\mu}}{I^{\mu}}\left(\sigma_{\max }-\sigma_{\min }\right)+\sigma_{\min }, \quad i=0,1,2, \ldots, I$

where $\sigma_{\min }$ and $\sigma_{\max }$ are the limits of $\sigma$, and $\mu$ is a positive real number called nonlinear modulation index. The value of $\mu$ controls the decreasing rate of $\sigma$. It is obvious that $\sigma$ is the same for all the seeds at a certain iteration. The parameters $\sigma_{\min }, \sigma_{\max }$ and $\mu$ are defined by the user.

\section{Step C: Competitive Exclusion}

Due to their invasive nature, the good weeds survive while the bad weeds are eliminated. So, the population size $W$ is restricted according to a maximum allowed number of weeds $P(W \leq P)$, which is user-defined. In order to determine which weeds are going to survive and which ones are going to be eliminated at the end of each $i$-th iteration, the fitness function is calculated for every seed being inside the search space, while seeds dispersed out of bounds are assigned a very high fitness value (penalty value). The fitness values of the weeds have already been calculated in the previous iteration. Then, all the members of the colony (i.e., weeds and dispersed seeds) are sorted according to their fitness values and the $P$ ones with the lowest fitness values survive and thus are able to produce seeds at the next iteration, while the rest of the sorted members are deleted. It is obvious that seeds dispersed outside the search space are very likely to be deleted due to their high fitness value. In this way, only weeds within the bounds survive.

\section{Termination Criterion}

The algorithm terminates when the maximum number of iterations $I$ is reached.

The above-described process seems to be effective. However, the performance of the IWO method is limited by the consideration of uniformly distributed initial positions of weeds inside the search space at the beginning of the method $(i=0)$. The uniform distribution is not always the best way to define the initial positions, especially in cases of few weeds distributed in an extensive $N$-dimensional search space where $N$ is a large number. In those cases, the uniform distribution 
may not be efficient enough to explore interesting regions of the search space. In the TI-IWO method, the weed positions are initialized in a different and more efficient way, as shown below.

\section{TAGUCHI'S OPTIMIZATION}

The Taguchi's Optimization (TO) is a non-stochastic method that exploits the beneficial properties of orthogonal arrays. A detailed description of TO and several of its applications in electromagnetics are given in [16].

The TO algorithm starts by selecting a proper orthogonal array $\mathrm{OA}(E, N, L, t)$, where $E$ is the number of array rows, $N$ is the number of array columns (i.e., the number of parameters to be optimized), $L$ is the number of levels, and $t$ is the strength. The $\mathrm{OA}(E, N, 3,2)$ (where $L=3$ and $t=2$ ) has been proved to be efficient for many problems [17]-[19], and thus this type of $\mathrm{OA}$ is utilized below. Each $e$-th row $(e=1,2, \ldots, E)$ of the OA corresponds to an experiment, which actually is a certain combination of $N$ values given respectively to the $N$ parameters. Due to the three levels considered here, three respective values $l v_{1}, l v_{2}$ and $l v_{3}$ are available for each parameter. The calculation of these values is described below.

For each $i$-th iteration, the following five steps are applied:

\section{Step A: Calculation of Level Differences}

The level differences are calculated for each $n$-th parameter by the following expression:

$l d(n, i)=r r^{i-1} \cdot l d(n, 1), \quad n=1,2, \ldots, N$

where $r r$ is the decreasing rate defined by the user, and $l d(n, 1)$ is the initial level difference calculated for each $n$-th parameter as given below:

$l d(n, 1)=\frac{x_{\max }(n)-x_{\min }(n)}{L+1}, \quad n=1,2, \ldots, N$

where $x_{\min }(n)$ and $x_{\max }(n)$ are the boundaries of the $n$-th parameter. As the value of $r r$ increases, the TO algorithm achieves better near-optimal values but converges slower.

\section{Step B: Calculation of Level Values}

For $i=1$, the 2 nd-level value of each $n$-th parameter is extracted as follows:

$l v_{2}(n, 1)=\frac{x_{\max }(n)+x_{\min }(n)}{2}, \quad n=1,2, \ldots, N$

For $i>1$, the 2 nd-level value $l v_{2}(n, i)$ is set equal to the optimum value $x_{o p t}(n, i-1)$ of the $n$-th parameter derived from the previous iteration $(i-1)$. However, $l v_{2}(n, i)$ is not permitted to become equal to $x_{\min }(n)$ or $x_{\max }(n)$, because one of the other two levels will exceed the boundaries. To avoid such a case, if $x_{\text {opt }}(n, i-1)=x_{\min }(n)$ then $l v_{2}(n, i)=x_{\text {min }}(n)+l d(n, i)$, and if $x_{o p t}(n, i-1)=x_{\max }(n)$ then $l v_{2}(n, i)=x_{\text {max }}(n)-l d(n, i)$.

Then, the 1st-level and 3rd-level values are respectively derived by the following expressions:

$l v_{1}(n, i)=l v_{2}(n, i)-l d(n, i), \quad n=1,2, \ldots, N$

$l v_{3}(n, i)=l v_{2}(n, i)+l d(n, i), \quad n=1,2, \ldots, N$

If $l v_{1}(n, i)<x_{\min }(n)$ then $l v_{1}(n, i)=x_{\min }(n), \quad$ and $\quad$ if $l v_{3}(n, i)>x_{\max }(n)$ then $l v_{3}(n, i)=x_{\max }(n)$. In this way, the 1 st-level and 3 rd-level values are kept within the boundaries.

\section{Step C:Fitness Function Estimation}

The fitness function $f i t(e, i)$ is estimated for each $e$-th experiment $(e=1,2, \ldots, E)$ and is converted to $\mathrm{dB}$ as shown below:

$$
\eta(e, i)=-20 \log \text { fit }(e, i), \quad e=1,2, \ldots, E
$$

\section{Step D:Construction of Response Table}

The impact of each level of each parameter is expressed by the average fitness in $\mathrm{dB}$, which is extracted by the following expression:

$\bar{\eta}(l, n, i)=\frac{L}{E} \sum_{e, O A(e, n)=l} \eta(e, i), l=1,2,3 \& n=1,2, \ldots, N$

The above average values are stored to a $3 \times N$ table called response table.

\section{Step E: Selection of the Optimum Values}

The optimum value $x_{\text {opt }}(n, i)$ of each $n$-th parameter is represented by one of the level values $l v_{1}(n, i), l v_{2}(n, i)$ and $l v_{3}(n, i)$, which corresponds to the highest value among $\bar{\eta}(1, n, i), \bar{\eta}(2, n, i)$ and $\bar{\eta}(3, n, i)$.

\section{Termination Criterion}

The optimization process terminates at the end of the $i$-th iteration when all ratios $l d(n, i) / l d(n, 1) \quad(n=1,2, \ldots, N)$ become less than a desired value defined by the user.

\section{Modifications to the Taguchi's Optimization Algorithm}

Some modifications are applied in order to decrease the complexity of the TO algorithm:

(i) The logarithmic term in (8) restricts the applicability of the algorithm, since only positive fitness values are allowed. To handle either positive or negative fitness values, fit $(e, i)$ 
is estimated for each $e$-th experiment $(e=1,2, \ldots, E)$, as mentioned above in step $\mathrm{C}$, but is not converted to $\mathrm{dB}$ and therefore (8) is no longer needed. So, the response table is simply filled in step D with fitness sums calculated as follows:

$$
s(l, n, i)=\sum_{e, O A(e, n)=l} f i t(e, i), l=1,2,3 \& n=1,2, \ldots, N
$$

The absence of logarithmic conversions and average fitness calculations decreases the complexity of the TO algorithm and saves computational time.

(ii) The use of fitness sums instead of average logarithmic fitness values has an impact on the selection of the optimum value $x_{\text {opt }}(n, i)$. This value is represented by one of the levels $l v_{1}(n, i), \quad l v_{2}(n, i)$ and $l v_{3}(n, i)$ that corresponds to the minimum sum among $s(1, n, i), s(2, n, i)$ and $s(3, n, i)$, and not to the largest $\bar{\eta}(l, n, i)$, as defined in the original TO algorithm.

(iii) The last modification concerns the termination criterion. The optimization process terminates at the end of the $i$-th iteration when all $l d(n, i)(n=1,2, \ldots, N)$ become less than a desired value defined by the user.

\section{TAGUChI INITIALIZED INVASIVE WEED OPTIMIZATION}

As mentioned above, in the conventional IWO, the initial weed positions are uniformly distributed inside the search space, but this type of distribution is not always efficient, especially when few weeds are distributed in a space defined by a large number of dimensions (i.e., a large number $N$ of parameters to be optimized). The TI-IWO method proposed in the present paper has the same structure as the conventional IWO method but employs the TO method to initialize effectively the positions of the weeds.

In the beginning of the TI-IWO method, a proper orthogonal array $\mathrm{OA}(E, N, 3,2)$ is selected according to the number $N$ of parameters to be optimized. As described in the previous section, each $e$-th row $(e=1,2, \ldots, E)$ of the OA is called an experiment and is a certain combination of $N$ values given respectively to the $N$ parameters. It is obvious that an experiment is the same as a weed in the IWO. Therefore, in the TI-IWO method, the number of weeds is not user-defined but depends on the number of rows $E$ of the OA. In addition, the initial position of each $e$-th weed is not distributed randomly inside the search space but depends on the level values of the $e$-th row of the OA. Then, the TO algorithm is applied only for one iteration (i.e., the five steps of the TO algorithm are applied only once) in order to derive an optimum position $x_{\text {opt }}$. The $E$ initial positions and the optimum one extracted by these positions compose a population of $E+1$ weeds $(W=E+1)$, which are dispersed inside the search space according to the iterative process of the IWO algorithm.

The superiority of the TI-IWO over the conventional IWO method can be confirmed by making a comparison between the two methods in terms of convergence. This comparison is shown below.

\section{V.FITNESS FUNCTION DEFINITION}

The antenna array design studied here is an inherently multi-objective problem, since several requirements must be simultaneously satisfied. On the other hand, all the optimization methods described above aim at finding the nearglobal minimum of a single mathematical function fit called fitness function. Therefore, fit must be formed as a linear combination of terms, which are defined according to the respective design requirements. When fit reaches its nearglobal minimum at the end of the optimization process, all the terms that compose fit find their respective minimum values and thus all the requirements are finally satisfied.

In the first two cases, two linear arrays of $M$ isotropic sources are optimized. The optimization process is required (i) to maximize the directional gain $G_{p}$ that corresponds to the peak of the main lobe, (ii) to tilt the main lobe by a desired angle $\Delta \theta_{\text {des }}$ defined by the desired direction of the main lobe and the normal to the array axis direction, and (iii) to prevent the directional gain inside a desired angular sector from becoming less than a desired value $g_{\text {des }}$ in relation to $G_{p}$ (desired null filling level). By taking into account the above requirements, the fitness function can be defined as follows:

$$
\begin{aligned}
f i t= & -b c_{1} G_{p}+b c_{2}\left|\Delta \theta_{a c t}-\Delta \theta_{d e s}\right| \\
& +b c_{3}\left[g_{d e s}-\min \left(g_{a c t}, g_{d e s}\right)\right]
\end{aligned}
$$

where $\Delta \theta_{a c t}$ is the actual tilting angle, $g_{a c t}$ is the minimum value of directional gain in relation to $G_{p}$ (i.e., the actual null filling level) inside the desired angular sector, and finally $b c_{1}$, $b c_{2}$ and $b c_{3}$ are positive coefficients used to balance the minimization of the three terms given in (11). Due to the third term in (11), values of $g_{a c t}$ greater than $g_{\text {des }}$ do not cause further minimization of fit, since the null filling requirement has already been satisfied. In the first two cases, $2 M-2$ parameters need to be optimized $(N=2 M-2)$ and these are the distances and phase differences between adjacent isotropic sources.

In the next two cases, two linear arrays of $M$ collinear wire dipoles in front of a mast are optimized. The optimization process has to satisfy the three requirements mentioned in the previous paragraph and also a fourth one concerning the impedance matching condition for every dipole. By taking into account all these requirements, the fitness function can be defined as given below:

$$
\begin{aligned}
f i t= & -b c_{1} G_{p}+b c_{2}\left|\Delta \theta_{a c t}-\Delta \theta_{d e s}\right| \\
& +b c_{3}\left[g_{\text {des }}-\min \left(g_{a c t}, g_{d e s}\right)\right]+b c_{4} \sum_{m=1}^{M}\left(S W R_{m}-1\right)
\end{aligned}
$$


where $b c_{4}$ is a positive coefficient that has the same meaning as $b c_{1}, b c_{2}$ and $b c_{3}$. Also, $S W R_{m}$ is the $S W R$ of the $m$-th dipole of the array $(m=1,2, \ldots, M)$ and is calculated by the expression:

$$
S W R_{m}=\frac{1+\left|r_{m}\right|}{1-\left|r_{m}\right|}
$$

where $\left|r_{m}\right|$ is the absolute value of the complex reflection coefficient $r_{m}$ at the input of the $m$-th dipole. The reflection coefficient is extracted as follows:

$r_{m}=\frac{Z_{m}-Z_{o}}{Z_{m}+Z_{o}}$

where $Z_{m}$ is the input impedance of the $m$-th dipole and $Z_{o}=50 \Omega$ is the characteristic impedance of the feeding lines. As $Z_{m}$ tends to reach $Z_{o}$ and thus satisfy the impedance matching condition, $S W R_{m}$ deceases and tends to unity. The values of $G_{p}, \Delta \theta_{a c t}, g_{a c t}$ and $Z_{m}(m=1,2, \ldots, M)$ needed to estimate fit from (12) are extracted by applying the MoM. In these two cases, $2 M+1$ parameters need to be optimized $(N=2 M+1)$ and these are the mast length, the dipole length (same for all the dipoles), the distance between the dipole array and the mast, and finally the distances between closer ends and phase differences of adjacent dipoles. The array is considered to be centered along the length of the mast.

\section{COMParative CONVERGEnce Results}

A comparison between the TI-IWO and the conventional IWO was made in terms of convergence. Each method was applied to optimize a linear array of 8 isotropic sources and executed 20 times with $n s_{\min }=0, n s_{\max }=5, \quad \sigma_{\min }=0$, $\sigma_{\max }=0.5, \mu=2.5$ and $I=5000 \quad$ (5000 iterations per execution). For $M=8,14$ parameters $(N=14)$ need to be optimized, i.e., 7 distances and 7 phase differences between adjacent sources. Therefore, an orthogonal array $\mathrm{OA}(81,14,3,2)$ must be utilized by the TI-IWO method, which means that a population of 82 weeds $(W=81+1)$ must be employed by the method. In order to have a fair comparison, the conventional IWO method must use the same population size.

The optimization process is applied for maximum $G_{p}$, $\Delta \theta_{d e s}=2^{\circ}$, and $g_{\text {des }}=-20 \mathrm{~dB}$ inside a $30^{\circ}$ sector from $90^{\circ}$ to $120^{\circ}$. The fitness function that takes into account the above requirements is defined by (11). This function is to be minimized by both methods. By executing the TI-IWO or the IWO method twenty times, twenty respective fitness values are recorded at every $i$-th iteration. These values are used to extract the average fitness of the $i$-th iteration. The variation of the average fitness with respect to the number of iterations represents the average convergence of the optimization method. The graphical representation of this variation is called average convergence graph and is extracted for both the TIIWO and IWO methods. These graphs are shown in Fig. 1. It is obvious that the fitness values achieved by the TI-IWO method start from lower levels and thus are lower than the values achieved by the conventional IWO method at every iteration. In addition, the IWO method needs notably more iterations to reach a certain fitness value than the TI-IWO method.

As mentioned in section IV, the difference between the two methods lies in the fact that the TI-IWO makes use of the TO method, while the IWO does not. However, since the TO method is applied in the beginning of the TI-IWO only for one iteration and thus is rapidly completed, it is obvious that the IWO and TI-IWO need almost the same computational time to complete the same number of iterations.

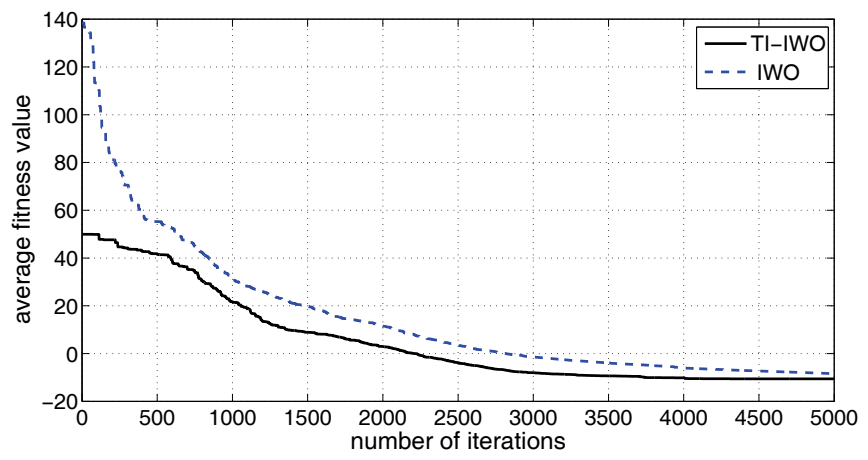

Fig. 1. Comparative convergence graphs of TI-IWO and conventional IWO.

\section{ANTENNA ARRAy OPTIMIZATION EXAMPLES}

The TI-IWO algorithm was applied to four cases of linear array optimization. A uniform-amplitude excitation distribution is considered in every case. The first two cases concern a theoretical aspect of linear array design and therefore the arrays are considered to be composed respectively of 8 (case 1) and 16 (case 2) isotropic sources. In these cases, the optimization is performed for maximum $G_{p}$, $\Delta \theta_{\text {des }}=2^{\circ}$, and $g_{\text {des }}=-20 \mathrm{~dB}$ inside a sector from $90^{\circ}$ to $120^{\circ}$, which are achieved by minimizing the fitness function given in (11). Since $G_{p}$ is required to be maximized without reaching any desired value, two reference values of directional gain are calculated in order to be used for comparison with $G_{p}$. These values are: (i) the maximum directional gain $G_{b p}$ of a broadside linear array (i.e., array without main lobe tilting, $\Delta \theta_{\text {des }}=0^{\circ}$ ) composed respectively of 8 (for case 1 ) and 16 (for case 2) isotropic sources with equal inter-element distances $d$ and equal excitation phases, and without the requirement for null filling, and (ii) the maximum directional gain $G_{t p}$ of a linear array composed respectively of 8 (for case 1) and 16 (for case 2) isotropic sources with equal interelement distances $d$ and equal excitation phase differences between adjacent sources given by the expression 
$\Delta \phi=\frac{2 \pi}{\lambda} d \sin \left(\Delta \theta_{d e s}\right)$

where $\Delta \theta_{\text {des }}=2^{\circ}$, and finally without the requirement for null filling.

The rest two cases concern realistic applications of linear array design and thus the arrays are considered to be composed respectively of 8 (case 3 ) and 16 (case 4) collinear wire dipoles in front of a mast. In these cases, the optimization is performed for maximum $G_{p}, \Delta \theta_{\text {des }}=2^{\circ}, g_{\text {des }}=-20 \mathrm{~dB}$ inside a sector from $90^{\circ}$ to $120^{\circ}$, and impedance matching of each dipole, which are achieved by minimizing the fitness function given in (12).

In all the cases, the TI-IWO algorithm is applied with $n s_{\text {min }}=0, n s_{\text {max }}=5, \sigma_{\text {min }}=0, \sigma_{\text {max }}=0.5$ and $\mu=2.5$. In cases 1 and 3 , where $M=8,14$ and 17 parameters need to be optimized, respectively. Therefore, the TI-IWO algorithm must use respectively $\mathrm{OA}(81,14,3,2)$ and $\mathrm{OA}(81,17,3,2)$, which results for both cases in a population of 82 weeds $(W=81+1)$. Also, the algorithm terminates after 5000 iterations $(I=5000)$. In cases 2 and 4 , where $M=16,30$ and 33 parameters must be optimized, respectively. Therefore, the TIIWO algorithm must use respectively $\mathrm{OA}(81,30,3,2)$ and $\mathrm{OA}(81,33,3,2)$, which implies again that $W=82$ for both cases. Due to the large number of optimization parameters in cases 2 and 4, 10000 iterations $(I=10000)$ are used to complete the execution of the algorithm.

\section{Case 1}

The radiation pattern of a linear array composed of $M$ isotropic sources with uniform-amplitude excitation distribution, equal inter-element distances $d$ and equal excitation phase differences $\Delta \phi$ between adjacent sources is derived from the absolute value of the array factor $A F$, which is given below:

$$
A F(\theta)=\sum_{m=0}^{M-1} \exp \left[j m\left(\frac{2 \pi}{\lambda} d \cos \theta+\Delta \phi\right)\right]
$$

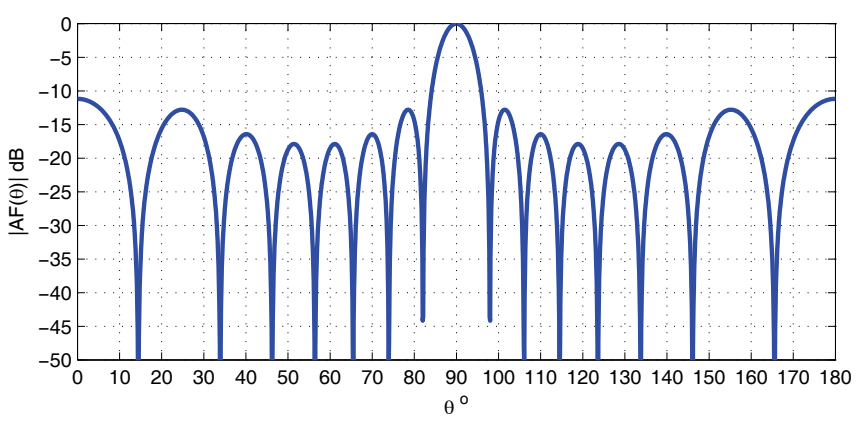

Fig. 2. Radiation pattern of a linear array composed of 8 isotropic sources with equal inter-element distances $d=0.904 \lambda$ and equal excitation phases $\left(G_{b p}=11.18 \mathrm{~dB}\right)$
By setting $\Delta \phi=0^{\circ}$ (equal phases), the array produces a broadside radiation pattern $\left(\Delta \theta_{d e s}=0^{\circ}\right)$. By assuming $M=8$ and by neglecting the requirement for null filling, the maximum directional gain $G_{b p}$ can be estimated by simply varying the value of $d$ and is found equal to $11.18 \mathrm{~dB}$ when $d=0.904 \lambda$. The respective radiation pattern is shown in Fig. 2. By neglecting again the requirement for null filling, the same array is studied assuming $\Delta \theta_{d e s}=2^{\circ}$. The main lobe tilting can be achieved by applying the same excitation phase difference $\Delta \phi$ between any adjacent sources. The value of $\Delta \phi$ is calculated from (15). Therefore, the maximum directional gain $G_{t p}$ can be estimated by simply varying the value of $d$ and is found equal to $11.07 \mathrm{~dB}$ when $d=0.879 \lambda$. This value of $d$ corresponds to $\Delta \phi=11.04^{\circ}$ and the respective radiation pattern is shown in Fig. 3.

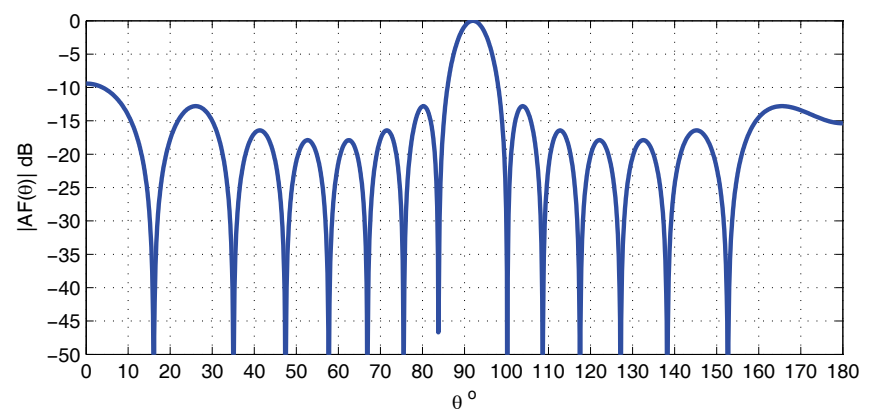

Fig. 3. Radiation pattern of a linear array composed of 8 isotropic sources with equal inter-element distances $d=0.879 \lambda$ and equal excitation phase differences $\Delta \phi=11.04^{\circ}$ between adjacent sources $\left(G_{t p}=11.07 \mathrm{~dB}\right)$.

TABLE I

ElEMENT Positions AND EXCITATION PHASES OF A NEAR-OPTIMAL LINEAR ARRAY COMPOSED OF 8 ISOTROPIC SOURCES

\begin{tabular}{ccc}
\hline Element & Position $(\lambda)$ & Phase (degrees) \\
\hline 1 & 0 & 0 \\
2 & 0.774 & 20.44 \\
3 & 2.726 & 20.26 \\
4 & 3.584 & 44.84 \\
5 & 4.470 & 44.52 \\
6 & 5.379 & 61.16 \\
7 & 6.252 & 71.80 \\
8 & 7.042 & 85.39 \\
\hline
\end{tabular}

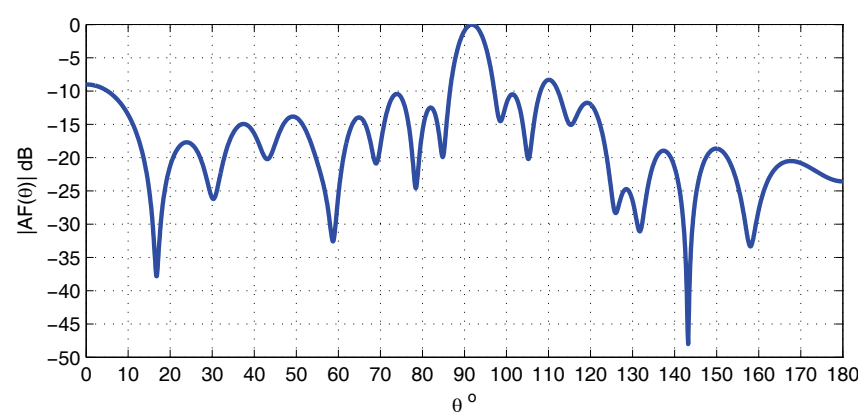

Fig. 4. Radiation pattern of a near-optimal linear array composed of 8 isotropic sources $\left(G_{p}=10.90 \mathrm{~dB}, \Delta \theta_{a c t}=1.9^{\circ}, g_{a c t}=-20.22 \mathrm{~dB}\right)$.

Finally, the TI-IWO algorithm is applied to the antenna 
array. The positions and phases of the array elements derived from the optimization process are presented in Table I. The radiation characteristics of the near-optimal array are $G_{p}=10.90 \mathrm{~dB}, \Delta \theta_{a c t}=1.9^{\circ}$, and $g_{a c t}=-20.22 \mathrm{~dB}$ inside the angular sector from $90^{\circ}$ to $120^{\circ}$, while the corresponding radiation pattern is shown in Fig. 4. The values of $\Delta \theta_{a c t}$ and $g_{a c t}$ are very close respectively to $\Delta \theta_{\text {des }}$ and $g_{d e s}$, while the optimal value of $G_{p}$ seems to be decreased only by $0.28 \mathrm{~dB}$ and $0.17 \mathrm{~dB}$ compared respectively to $G_{b p}$ and $G_{t p}$.

\section{Case 2}

The whole procedure is repeated considering a linear array composed of 16 isotropic sources $(M=16)$. By assuming $\Delta \phi=0^{\circ}$ for all the array elements $\left(\Delta \theta_{\text {des }}=0^{\circ}\right)$ and neglecting the null filling requirement, the maximum gain $G_{b p}$ is calculated equal to $14.41 \mathrm{~dB}$ when $d=0.948 \lambda$. The radiation pattern is shown in Fig. 5. By applying the same excitation phase difference $\Delta \phi$ between any adjacent sources in order to tilt the main lobe by $2^{\circ}\left(\Delta \theta_{d e s}=2^{\circ}\right)$ and neglecting again the null filling requirement, the maximum gain $G_{t p}$ is calculated equal to $14.36 \mathrm{~dB}$ when $d=0.919 \lambda$. This value of $d$ corresponds to $\Delta \phi=11.55^{\circ}$ and the respective radiation pattern is shown in Fig. 6.

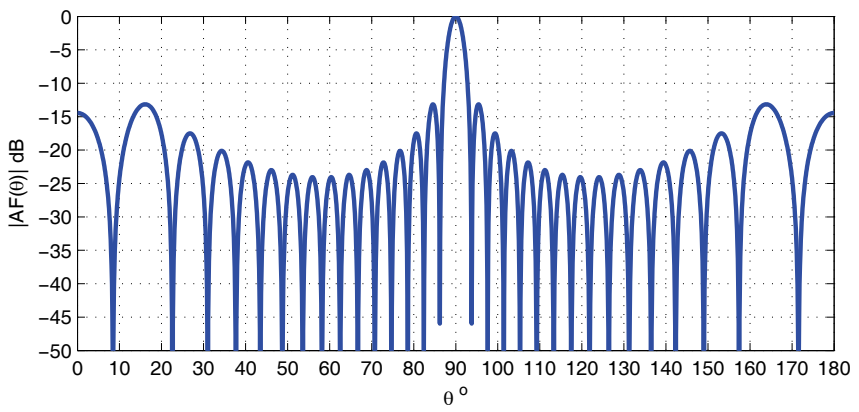

Fig. 5. Radiation pattern of a linear array composed of 16 isotropic sources with equal inter-element distances $d=0.948 \lambda$ and equal excitation phases $\left(G_{b p}=14.41 \mathrm{~dB}\right)$

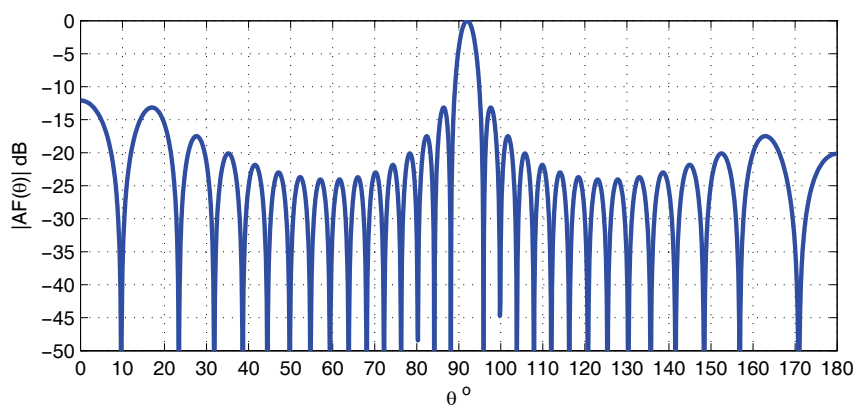

Fig. 6. Radiation pattern of a linear array composed of 16 isotropic sources with equal inter-element distances $d=0.919 \lambda$ and equal excitation phase differences $\Delta \phi=11.55^{\circ}$ between adjacent sources $\left(G_{t p}=14.36 \mathrm{~dB}\right)$.

Then, the antenna array is optimized by the TI-IWO algorithm. The positions and phases of the array elements resulted from the optimization process are presented in Table II. The radiation characteristics of the near-optimal array are $G_{p}=14.36 \mathrm{~dB}, \Delta \theta_{a c t}=1.9^{\circ}$, and $g_{a c t}=-18.89 \mathrm{~dB}$ inside the angular sector from $90^{\circ}$ to $120^{\circ}$, while the respective radiation pattern is displayed in Fig. 7. It seems that $\Delta \theta_{\text {act }}$ is very close to $\Delta \theta_{\text {des }}$, and $g_{a c t}$ totally satisfies the null filling requirement. In addition, the optimal value of $G_{p}$ is not downgraded at all compared to $G_{t p}$ and seems to be decreased only by $0.05 \mathrm{~dB}$ compared to $G_{b p}$.

TABLE II

ELEMENT POSITIONS AND EXCITATION PHASES OF A NEAR-OPTIMAL LINEAR

\begin{tabular}{ccc}
\multicolumn{3}{c}{ ARRAY COMPOSED OF 16 ISOTROPIC SOURCES } \\
\hline Element & Position $(\lambda)$ & Phase (degrees) \\
\hline 1 & 0 & 0 \\
2 & 1.778 & 62.37 \\
3 & 2.630 & 77.21 \\
4 & 4.358 & 68.51 \\
5 & 6.291 & 61.47 \\
6 & 7.063 & 110.82 \\
7 & 8.242 & 117.19 \\
8 & 9.106 & 138.65 \\
9 & 9.986 & 151.15 \\
10 & 11.945 & 139.73 \\
11 & 12.725 & 156.89 \\
12 & 13.639 & 168.40 \\
13 & 15.512 & 193.45 \\
14 & 16.230 & 217.97 \\
15 & 17.030 & 243.00 \\
16 & 17.920 & 249.20 \\
\hline
\end{tabular}

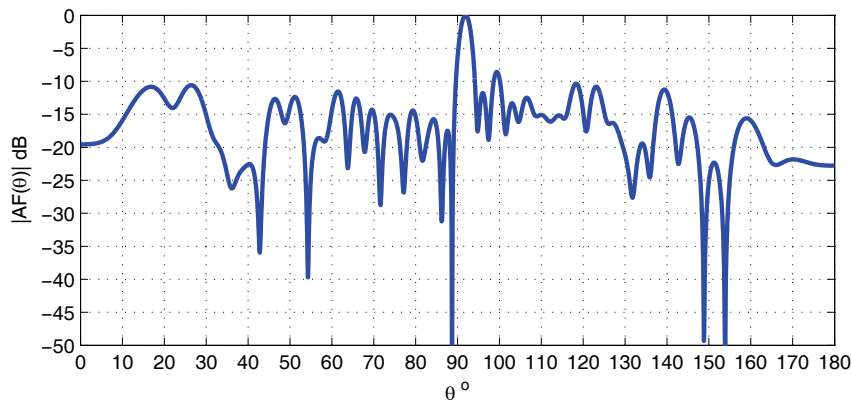

Fig. 7. Radiation pattern of a near-optimal linear array composed of 16 isotropic sources $\left(G_{p}=14.36 \mathrm{~dB}, \Delta \theta_{a c t}=1.9^{\circ}, g_{a c t}=-18.89 \mathrm{~dB}\right)$.

\section{Case 3}

This case concerns a linear array of 8 collinear wire dipoles in front of a mast. The MoM is used here as a full wave analysis engine that extracts the radiation characteristics of the array every time the TI-IWO algorithm needs to calculate fit from (12). Both the array geometry and phases derived from the optimization process as well as the $S W R$ values of the dipoles of the near-optimal array are presented in Table III. Also, the radiation pattern of the array is displayed in Fig. 8.

The results show that $\Delta \theta_{\text {act }}$ is very close to $\Delta \theta_{\text {des }}$, and $g_{a c t}$ totally satisfies the null filling requirement. In addition, the optimal value of $G_{p}$ is $2.24 \mathrm{~dB}$ over the value of $G_{p}$ of the respective 8 -source linear array. This difference is well explained by the radiation pattern of the dipole itself and the presence of the mast. Finally, the values of $S W R$ are close 
enough to unity and thus the impedance matching condition is well satisfied for every dipole.

TABLE III

GEOMETRY, EXCITATION PHASES AND SWR VALUES OF A NEAR-OPTIMAL ARRAY COMPOSED OF 8 COLLINEAR WIRE DIPOLES IN FRONT OF A MAST

\begin{tabular}{cccc}
\hline Dipole & $\begin{array}{c}\text { Position of Dipole } \\
\text { Center }(\lambda)\end{array}$ & $\begin{array}{c}\text { Phase } \\
(\text { degrees })\end{array}$ & SWR \\
\hline 1 & 0 & 0 & 1.11 \\
2 & 1.678 & 343.08 & 1.08 \\
3 & 3.205 & 18.62 & 1.13 \\
4 & 4.429 & 67.38 & 1.09 \\
5 & 5.943 & 1.05 & 1.08 \\
6 & 6.815 & 64.99 & 1.17 \\
7 & 8.523 & 99.70 & 1.15 \\
8 & 9.667 & 125.96 & 1.14 \\
\hline
\end{tabular}

Dipole Length $=0.467 \lambda$

Mast Length $=12.956 \lambda$

Distance between dipoles and mast $=0.125 \lambda$

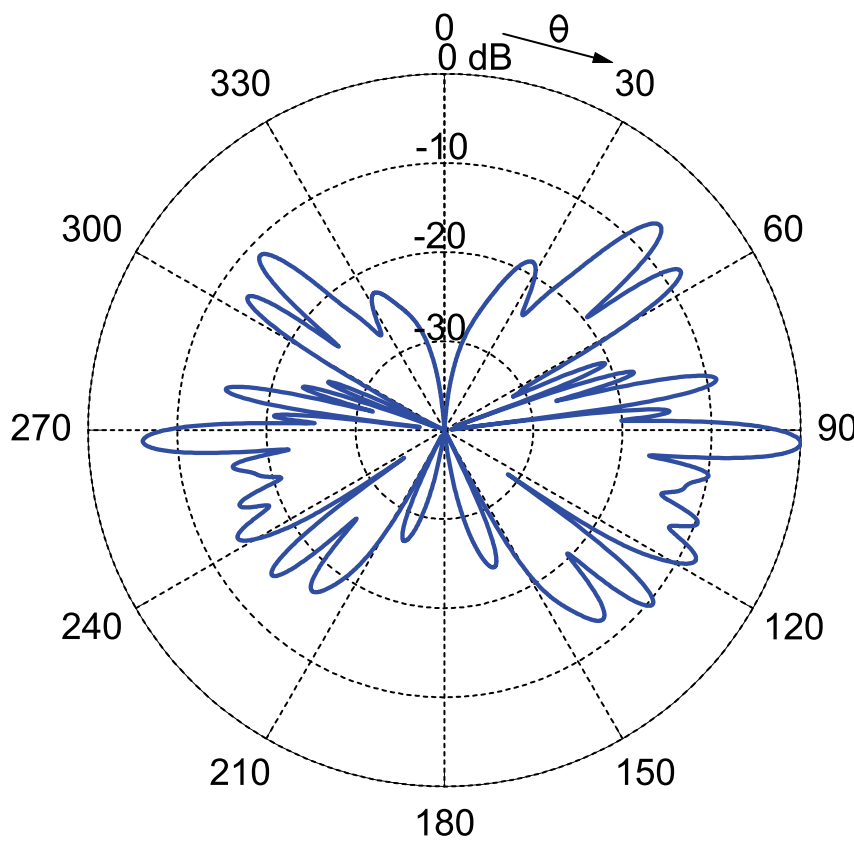

Fig. 8. Radiation pattern of a near-optimal array composed of 8 collinear wire dipoles in front of a mast $\left(G_{p}=13.14 \mathrm{~dB}, \Delta \theta_{a c t}=2.1^{\circ}, g_{a c t}=-16.86 \mathrm{~dB}\right)$.

\section{Case 4}

In this case, the optimization process is applied to a linear array of 16 collinear wire dipoles in front of a mast. The array geometry, the excitation phases and the $S W R$ values of the dipoles of the near-optimal array are presented in Table IV. Also, the radiation pattern of the array is shown in Fig. 9.

The results show that $\Delta \theta_{a c t}$ and $g_{a c t}$ are very close respectively to $\Delta \theta_{\text {des }}$ and $g_{\text {des }}$. In addition, the optimal value of $G_{p}$ is $2.25 \mathrm{~dB}$ over the value of $G_{p}$ of the respective 16source linear array. This difference can be explained in the same way as in the previous case. Finally, the $S W R$ values show that the impedance matching condition is well satisfied for all the dipoles of the array.
TABLE IV

GEOMETRY, EXCITATION PHASES AND SWR VALUES OF A NEAR-OPTIMAL ARRAY COMPOSED OF 16 COLLINEAR WIRE DIPOLES IN FRONT OF A MAST

\begin{tabular}{cccc}
\hline Dipole & $\begin{array}{c}\text { Position of Dipole } \\
\text { Center }(\lambda)\end{array}$ & $\begin{array}{c}\text { Phase } \\
\text { (degrees) }\end{array}$ & SWR \\
\hline 1 & 0 & 0 & 1.25 \\
2 & 1.778 & 14.80 & 1.25 \\
3 & 3.386 & 52.37 & 1.24 \\
4 & 5.157 & 43.18 & 1.26 \\
5 & 6.681 & 32.18 & 1.27 \\
6 & 7.931 & 112.12 & 1.32 \\
7 & 9.078 & 78.04 & 1.27 \\
8 & 10.511 & 133.60 & 1.27 \\
9 & 11.944 & 129.62 & 1.26 \\
10 & 13.787 & 107.32 & 1.17 \\
11 & 14.740 & 161.65 & 1.23 \\
12 & 15.782 & 196.31 & 1.29 \\
13 & 17.333 & 171.82 & 1.20 \\
14 & 18.292 & 203.52 & 1.24 \\
15 & 19.926 & 214.83 & 1.26 \\
16 & 21.239 & 264.64 & 1.29 \\
\hline \multicolumn{4}{c}{ Dipole Length $=0.473 \lambda$} \\
\end{tabular}

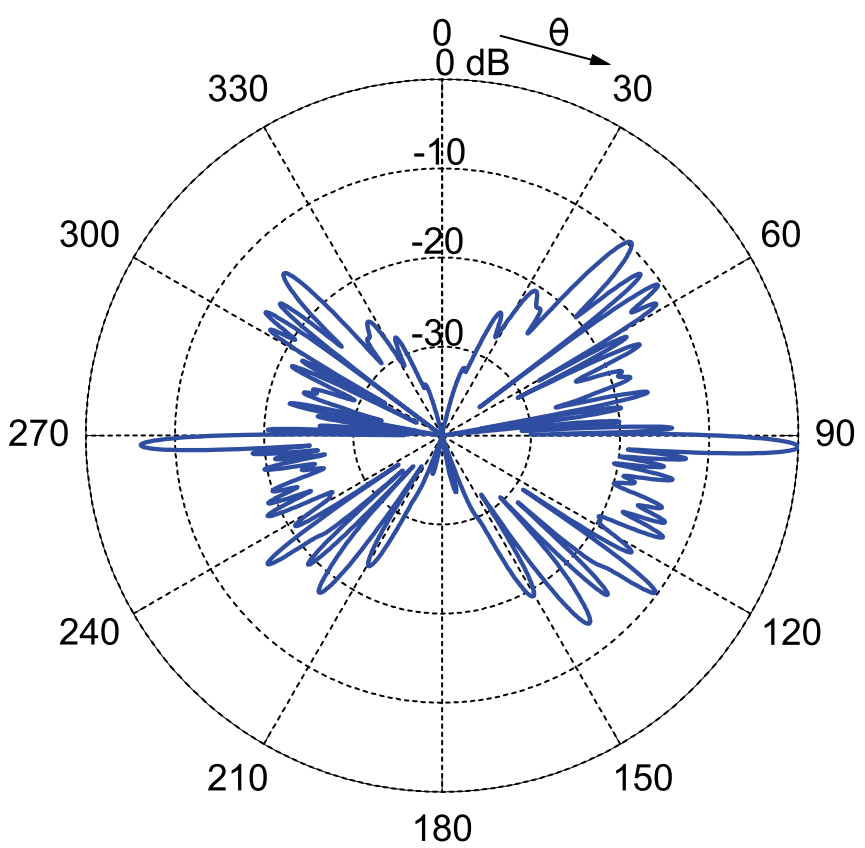

Fig. 9. Radiation pattern of a near-optimal array composed of 16 collinear wire dipoles in front of a mast $\left(G_{p}=16.61 \mathrm{~dB}, \Delta \theta_{a c t}=1.9^{\circ}, g_{a c t}=-20.45 \mathrm{~dB}\right)$.

\section{VIII.CONCLUSION}

A powerful optimization method suitable for antenna arrays has been presented. The method, called TI-IWO, is a novel IWO variant initialized by applying the Taguchi's Optimization method. Due to the particular type of initialization, the TI-IWO starts from lower fitness values and thus results in better solutions than the conventional IWO, as shown by a comparison in terms of convergence between these two methods.

The proposed method has been applied to optimize basestation antenna arrays suitable for broadcasting applications. Therefore, the optimization process has been performed under 
requirements for maximum gain, main lobe tilting and null filling, while in realistic cases of collinear wire dipole arrays an additional requirement concerning the impedance matching of all the dipoles has to be satisfied as well. In the cases studied here, the requirements for main lobe tilting, null filling and impedance matching seem to be well satisfied. In addition, the optimal value of gain achieved by the TI-IWO algorithm is almost equal to or slightly lower than the gain achieved without requirements for main lobe tilting and null filling. The slight decrease in gain can be considered as a small cost paid to simultaneously satisfy all the other requirements. Due to its excellent performance, the TI-IWO algorithm finally seems to be very useful in many applications of broadcasting technology.

\section{REFERENCES}

[1] I. Kosalay, "Estimation of RF electromagnetic levels around TV broadcast antennas using fuzzy logic," IEEE Trans. Broadcast., vol. 56, no. 1, pp. 36-43, Mar. 2010.

[2] M. Milojević, G. Del Galdo, N. Song, M. Haardt, and A. Heuberger, "Impact of the receive antenna arrays on spatio-temporal availability in satellite-to-indoor broadcasting," IEEE Trans. Broadcast., vol. 56, no. 2 , pp. 171-183, June 2010

[3] P. Mousavi, M. Fakharzadeh, and S. Safavi-Naeini, "1K element antenna system for mobile direct broadcasting satellite reception," IEEE Trans. Broadcast., vol. 56, no. 3, pp. 340-349, Sept. 2010.

[4] F. J. Ares-Pena, J. A. Rodriguez-Gonzalez, E. Villanueva-Lopez, and S. R. Rengarajan, "Genetic algorithms in the design and optimization of antenna array patterns," IEEE Trans. Antennas Propagat., vol. 47, no. 3, pp. 506-510, Mar. 1999.

[5] J. Hirokawa, C. Yamazaki, and M. Ando, "Postwall waveguide slot array with cosecant radiation pattern and null filling for base station antennas in local multidistributed systems," Radio Science, vol. 37, no. 2, pp. VIC10/1-VIC10/7, Apr. 2002.

[6] K. Y. Hui and K. M. Luk, "Design of wideband base station antenna arrays for CDMA 800 and GSM 900 systems," Microw. Opt. Technol. Lett., vol. 39, no. 5, pp. 406-409, Dec. 2003.

[7] S.-I. Yamamoto, N. Hikino, J. Hirokawa, and M. Ando, "A post-wall waveguide slot array with a three-way power divider on a single-layer dielectric substrate," IEICE Trans. Communications, vol. E88-B, no. 4, pp. 1740-1742, 2005

[8] W. Shen and W. X. Zhang, "Pattern synthesis of non-symmetric tapered slotline antenna," Electronics Letters, vol. 42, no. 8, pp. 443-444, Apr. 2006.

[9] A. R. Mehrabian and C. Lucas, "A novel numerical optimization algorithm inspired from weed colonization," Ecological Informatics, vol. 1, pp. 355-366, 2006.

[10] A. R. Mallahzadeh, S. Es'haghi, and A. Alipour, "Design of an e-shaped MIMO antenna using IWO algorithm for wireless application at 5.8 GHz," Prog. Electromagn. Res., vol. 90, 187-203, 2009.

[11] S. Pal, A. Basak, S. Das, and A. Abraham, "Linear antenna array synthesis with invasive weed optimization algorithm," in Proc. 2009 Int Conf. Soft Computing and Pattern Recognition, Dec. 2009, pp. 161-166.

[12] S. Karimkashi and A. A. Kishk, "Invasive weed optimization and it features in electromagnetics," IEEE Trans. Antennas Propagat., vol. 58, no. 4, pp. 1269-1278, Apr. 2010

[13] F. M. Monavar and N. Komjani, "Bandwidth enhancement of microstrip patch antenna using jerusalem cross-shaped frequency selective surfaces by invasive weed optimization approach," Prog. Electromagn. Res., vol. 121, pp. 103-120, 2011.

[14] S. Karimkashi, A. A. Kishk, and D. Kajfez, "Antenna array optimization using dipole models for MIMO applications," IEEE Trans. Antennas Propagat., vol. 59, no. 8, pp. 3112-3116, Aug. 2011.

[15] R. C. Hansen, Moment Methods in Antennas and Scattering. Norwood, MA: Artech House, 1990.
[16] W. C. Weng, F. Yang, and A. Elsherbini, Electromagnetics and Antenna Optimization Using Taguchi's Method. San Rafael, CA: Morgan \& Claypool, 2007.

[17] W. C. Weng and C. Choi, "Optimal design of CPW slot antennas using Taguchi's method," IEEE Trans. Magnetics, vol. 45, no. 3, pp. 15421545, Mar. 2009.

[18] N. Sheng, C. Liao, W. Lin, L. Chang, Q. Zhang, and H. Zhou, "A hybrid optimized algorithm based on EGO and Taguchi's method for solving expensive evaluation problems of antenna design," Prog. Electromagn. Res. C, vol. 17, pp. 181-192, 2010.

[19] N. Nemri, A. Smida, R. Ghayoula, H. Trabelsi, and A. Gharsallah, "Phase-only array beam control using a Taguchi optimization method," in Proc. 11th Mediterranean Microwave Symposium (MMS), Sept. 2011, pp. $97-100$.

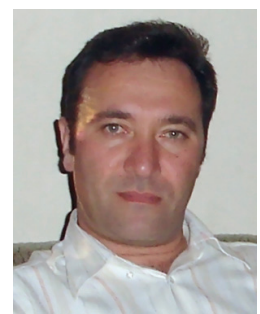

Zaharias D. Zaharis received the B.Sc. degree in Physics in 1987, the M.Sc. degree in Electronics Physics in 1994 and the Ph.D. degree in 2000 from Aristotle University of Thessaloniki. Also, in 2011 he obtained the Diploma degree in Electrical and Computer Engineering from the same university. Since 2002 he has been working in the administration of the telecommunications network at the Aristotle University of Thessaloniki.

His research interests include design and optimization of antenna arrays, design and optimization of microwave circuits and systems, mobile communications, RF measurements, radiowave propagation and electromagnetic scattering.

Dr. Zaharis is a member of the Greek Physics Society and the Technical Chamber of Greece.

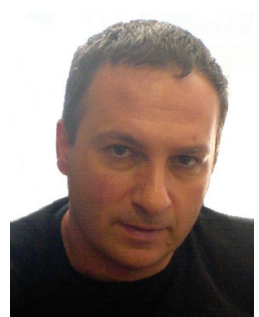

Pavlos I. Lazaridis was born in Larissa, Greece. He received the electrical engineering degree from Aristotle University of Thessaloniki, Greece, in 1990, the DEA degree in electronics from the Université Pierre \& Marie Curie, Paris, France, in 1992, and the Ph.D. degree in electronics and telecommunications from the Ecole Nationale Supérieure des Télécommunications (ENST), Paris, in 1996.

From 1989 to 1990 , he was with the Greek Public Power Corporation, Athens, Greece, where he worked on network reliability evaluation algorithms. From 1991 to 1996, he was involved with research on semiconductor lasers, wave propagation, and nonlinear phenomena in optical fibers for the Centre National d'Etudes des Télécommunications (CNET) and teaching at the ENST. In 1997, he became Head of the Antennas and Propagation Laboratory, TDF Metz (Télédiffusion de France / France Télécom research center), where he was involved with research in antenna coupling and radio coverage prediction algorithms for cellular mobile systems, DAB, and DVB-T broadcasting. From 1998 to 2002 he was with the European Patent Office (EPO) Rijswijk, The Netherlands, as a senior examiner in the field of Electronics-Telecommunications. He is currently teaching as a senior lecturer at the MSc course jointly organized by Alexander Technological Educational Institute of Thessaloniki, Greece, and Brunel University, West London.

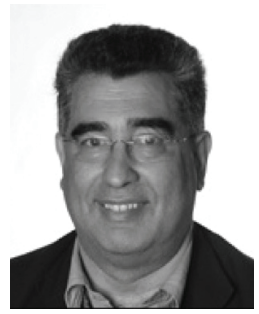

John Cosmas is a Professor of Multimedia Systems in the School of Engineering and Design at Brunel University in West London. He co-leads the Wireless Networks and Communications Research Centre, (http://www.brunel.ac.uk/sed/ece/research/wncc), is the course director of MSc Advanced Multimedia Design and 3D Technologies (http://www.brunel.ac.uk/sed/ece/courses/postgradua te/advanced-multimedia-design-and-3dTransactions on Broadcasting.

His research interests are concerned with the development of Multimedia Systems applied to Future of Broadcasting, the Future of Internet and 3D multimedia video/graphics design and the synergies between these technologies. He has participated in eleven EU-IST and two EPSRC funded research projects since 1986 and he has led three of these (CISMUNDUS, PLUTO, 3D MURALE). 


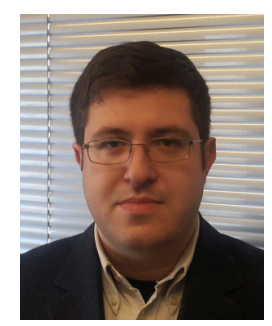

Christos Skeberis received an Engineering Diploma Degree in Electrical and Computer Engineering in 2010 from Aristotle University of Thessaloniki and currently is a Ph.D. candidate in the same university.

His research interests include design and optimization of antenna arrays, mobile communications systems, neural networks, machine learning, radiowave propagation and ionospheric phenomena.

Mr. Skeberis is a member of the Technical Chamber of Greece.

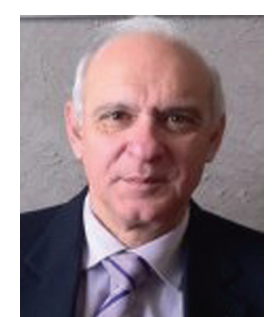

Thomas D. Xenos was born in Thessaloniki, Greece, in 1955. He received the Diploma in electrical engineering from the University of Patras, Patras, Greece, in 1978, and the Ph.D. degree in wireless communications from the Aristotle University of Thessaloniki, Thessaloniki, Greece, in 1991.

Since then, he joined the Department of Electrical and Computer Engineering, Aristotle University of Thessaloniki, where he is currently a Professor. He took part in many national and international projects. $\mathrm{He}$ is the author of over 85 scientific papers. His research interests include wireless communications, radiowave propagation, nonionizing electromagnetic radiation measurements, and electromagnetic compatibility. 